\title{
BMJ Global Health Integration of non-communicable disease and HIV/AIDS management: a review of healthcare policies and plans in East Africa
}

Olukemi Adeyemi, ${ }^{1}$ Mary Lyons, ${ }^{1}$ Tsi Njim, ${ }^{1}$ Joseph Okebe, ${ }^{1}$ Josephine Birungi, ${ }^{2}$ Kevin Nana, ${ }^{3}$ Jean Claude Mbanya, ${ }^{3}$ Sayoki Mfinanga, ${ }^{4}$ Kaushik Ramaiya, ${ }^{5}$ Shabbar Jaffar (D) , Anupam Garrib ${ }^{6}$

To cite: Adeyemi 0 , Lyons M, Njim T, et al. Integration of noncommunicable disease and HIV/ AIDS management: a review of healthcare policies and plans in East Africa. BMJ Global Health 2021;6:e004669. doi:10.1136/ bmjgh-2020-004669

Handling editor Seye Abimbola

Received 6 December 2020 Revised 27 March 2021 Accepted 2 April 2021
Check for updates

(c) Author(s) (or their employer(s)) 2021. Re-use permitted under CC BY-NC. No commercial re-use. See rights and permissions. Published by BMJ.

For numbered affiliations see end of article.

Correspondence to Dr Anupam Garrib; anupam.garrib@|stmed.ac.uk

\section{ABSTRACT}

Background Low-income and middle-income countries are struggling to manage growing numbers of patients with chronic non-communicable diseases (NCDs), while services for patients with HIV infection are well established. There have been calls for integration of HIV and NCD services to increase efficiency and improve coverage of NCD care, although evidence of effectiveness remains unclear. In this review, we assess the extent to which National HIV and NCD policies in East Africa reflect the calls for HIV-NCD service integration.

Methods Between April 2018 and December 2020, we searched for policies, strategies and guidelines associated with HIV and NCDs programmes in Burundi, Kenya, Rwanda, South Sudan, Tanzania and Uganda. Documents were searched manually for plans for integration of HIV and NCD services. Data were analysed qualitatively using document analysis. Results Thirty-one documents were screened, and 13 contained action plans for HIV and NCDs service integration. Integrated delivery of HIV and NCD care is recommended in high level health policies and treatment guidelines in four countries in the East African region; Kenya, Rwanda, Tanzania and Uganda, mostly relating to integrating NCD care into HIV programmes. The increasing burden of NCDs, as well as a move towards person-centred differentiated delivery of services for people living with HIV, is a factor in the recent adoption of integrated HIV and NCD service delivery plans. Both South Sudan and Burundi report a focus on building their healthcare infrastructure and improving coverage and quality of healthcare provision, with no reported plans for HIV and NCD care integration.

Conclusion Despite the limited evidence of effectiveness, some East African countries have already taken steps towards HIV and NCD service integration. Close monitoring and evaluation of the integrated HIV and NCD programmes is necessary to provide insight into the associated benefits and risks, and to inform future service developments.

\section{INTRODUCTION}

There are increasing calls for an integrated health system response to the management of HIV infection and the growing

\section{Key questions}

What is already known?

- There is a high prevalence of HIV and rapidly increasing prevalence of non-communicable diseases (NCDs) in low-income and middle-income countries (LMICs).

- There is an increased risk of NCDs among people living with HIV.

- The integration of services for HIV and NCDs may enable LMICs to expand coverage of healthcare for people living with NCDs and address risks of developing NCDs amongst people living with HIV.

- There is limited evidence of the benefits and potential risks associated with integration of HIV and NCD services.

What are the new findings?

- Some countries in East Africa have already initiated HIV and NCD service integration and included guidance to this effect in high level policies.

- The approaches to integration mainly include incorporating NCD prevention and control into existing HIV services.

- Integrating HIV and NCD services is seen as a strategy to contribute to achieving the Sustainable Development Goals and Universal Health Coverage.

What do the new findings imply?

- There is a need to monitor and evaluate the integration delivery of HIV and NCD services, to determine the impact of integration on the quality of care delivered and patient outcomes.

non-communicable disease (NCD) epidemics in low-income and middle-income countries, aimed at addressing poor coverage of NCD services and evidence of a high burden of multimorbidity in people living with HIV. ${ }^{12}$

However, evidence for the benefits of integration is limited. ${ }^{3}$ Indeed, integration may have a negative impact on progress that has been made in HIV treatment programmes. ${ }^{4-6}$ 
There is little available evidence on the cost-effectiveness of integration or its overall impact on health systems.

In this review, we assess the extent to which national policies and guidelines for HIV and NCDs care, specifically care for diabetes and hypertension, in selected East African countries reflect the movement towards integration of HIV and NCD services.

\section{The burden of HIV and NCDS}

HIV infection remains a major public health issue with continuing high HIV incidence. ${ }^{7}$ The increased availability and uptake of highly active antiretroviral therapy has resulted in a decline in HIV associated mortality and an increase in life expectancy, turning HIV into a chronic medical condition requiring linkage into and retention in care, and all the necessary resources to maintain care delivery over the patient's lifetime. ${ }^{28-10}$ In Africa, there are 15 million people on antiretroviral therapy who are at increased risk of developing NCDs due to ageing and the additional impact of long standing HIV infection and exposure to antiretroviral drugs. ${ }^{11}$

The global burden of NCDs has risen rapidly, causing 15 million premature deaths each year among people aged 30-69 years, with $85 \%$ of these deaths occuring in low-income and middle-income countries. ${ }^{12}$ NCD-related deaths are projected to increase at a higher rate in Africa compared with worldwide trends and could exceed deaths from infectious diseases by $2030 .^{12} 13$

Evidence on the prevalence of diabetes and hypertension in low-income and middle-income countries is limited, although it is accepted that there is considerable underdiagnosis, that few people are in regular care, and fewer still are well controlled on treatment. ${ }^{1415}$

\section{Healthcare provision for HIV/AIDS and NCDS}

The investment in HIV programmes has had both positive and negative effects on health systems in low-income and middle-income countries. It has strengthened health services through investment in physical infrastructure, laboratory capacity, health information systems, healthcare worker capacity development and promoting delivery of antenatal care, family planning and sexually transmitted infection (STI) management; but also diverted financial and human resources away from other health programmes. ${ }^{16}$ This is especially relevant where healthcare resources are severely constrained, and health systems are focused on managing acute infections.

Coverage of HIV services has recently improved with increasing numbers entering treatment programmes, being retained in care and achieving viral suppression. In Eastern and Southern Africa, antiretroviral therapy coverage was estimated at $67 \%$ with $87 \%$ of those on treatment having a suppressed viral load. ${ }^{17}$

In contrast, health service provision for NCDs in subSaharan Africa remains poor and evidence on adherence to treatment and retention in care is limited. ${ }^{18}$ Available evidence for both hypertension and diabetes suggests that retention in care deteriorates significantly after diagnosis and treatment initiation, to as low as less than $30 \%$ at 12 months. ${ }^{19}{ }^{20}$ In a systematic review published in 2007, covering sub-Saharan Africa, less than $30 \%$ of people diagnosed with hypertension were on drug treatment. ${ }^{14}$

\section{Integration of HIV/AIDS and NCD services}

Integrated service delivery refers to:

the organisation and management of health services so that people get the care they need, when they need it, in ways that are user-friendly, to achieve the desired results and provide value for money, $\mathrm{pg} 1 .^{21}$

There is a lack of large, good-quality rigorous studies able to provide the evidence to support integration as a preferred approach to service delivery in low-income and middle-income countries. ${ }^{32}$ Methods of integrating services range from 'adding' a service to an existing service platform to full integration of parallel services. ${ }^{3}$ 'Adding on' a service may be cost saving and may increase service utilisation, however, does not necessarily improve service delivery nor patient outcomes when compared with vertical programmes. Full integration of services may also reduce the knowledge and utilisation of 'special services' and may not result in any improvements in health status.

The integration of health services has been on the agenda since the establishment of primary healthcare in the 1980s, and more recently as a means to help achieve the Sustainable Development Goals and Universal Health Coverage. ${ }^{23-25}$

Several global HIV and NCD strategies encourage the integration of HIV and NCDs management. The most recent Joint United Nations Programme on HIV/AIDS (UNAIDS) targets include several on integration. ${ }^{24} 26-28$ Suggested reasons for recommending service integration include the epidemiological overlap between these conditions, with high prevalence of HIV and NCDs in many low-income and middle-income countries ${ }^{21222}$; the increased risk of NCDs and high health burden when the conditions occur together, ${ }^{1329}$ and the similar healthcare service provision strategies required to manage HIV and NCDs as chronic conditions. ${ }^{27}$

A further driver for HIV-NCD integration is the need for countries with a high HIV burden to develop efficient and cost-effective ways to deliver their HIV programmes as external funding for HIV programmes decreases. ${ }^{30} 31$

\section{Models of NCD-HIV service integration}

Several reviews have identified different models of HIVNCD service integration. ${ }^{4-632}$ These include NCD prevention and control incorporated into existing HIV services; HIV prevention and control added to primary healthcare already providing NCD services, and simultaneous introduction of integrated HIV and NCD services. ${ }^{32}$

\section{Benefits and risks of HIV-NCD integration}

HIV programmes have developed considerable systems innovations including decentralised care, task-shifting, counselling, community engagement, drugs and 
diagnostics procurement and treatment adherence support for the management of HIV as a chronic condition, all of which could now be used to inform the development of other high-quality chronic care services. ${ }^{83} 34$

Potential benefits of HIV-NCD integration for the health system and patients include a reduction in duplication and fragmentation of services, which would increase efficiency of resource use and help patients remain in care by reducing costs and inconvenience for patients with multiple morbidities. ${ }^{32}{ }^{34-36}$ Screening for NCDs within HIV care programmes can improve the identification of undiagnosed NCDs among patients living with HIV and also contribute to improved health outcomes. ${ }^{37-39}$

Possible disadvantages to the integration of HIV and NCD services include an increased workload for HIV or NCD programme staff which, particularly for HIV care, will require careful management. ${ }^{4}$ Successful integration of NCD care into HIV services will require an expansion of service provision overall, as well as significant systems reorganisation to allow documentation, drug delivery and healthcare worker support. ${ }^{40}$ HIV-NCD integration without appropriate resourcing runs the risk of reducing the quality of current HIV services without improving NCD care. There is also a risk that stigma may influence the uptake of services for NCD care. ${ }^{6}$

\section{Role of national health policies, strategies and plans}

National health policies, strategies and plans are the tools governments use to respond to the demands for change or improvement in health systems and healthcare delivery, providing direction and importantly financial support for change. ${ }^{41}$ Ideally, national health strategies, policies and plans, should be informed by robust evidence and used to create an efficient, effective health service.

\section{METHODS}

\section{Search strategy}

A purposeful web search for the most recent, publicly available general health policy statements, strategic plans and clinical guidelines covering the delivery of care for HIV and NCDs in the East African Community was undertaken. The East African Community was established in 1967, has a population of 145.5 million and comprises six countries: Burundi, Kenya, Rwanda, South Sudan, Tanzania and Uganda ${ }^{42}$ Kenya is a lower-middle-income country, while other countries in the community are classified as low-income countries. ${ }^{43}$

The searches were completed in December 2020 using advanced features in Google Scholar. No limits were applied. Keywords included Burundi, Kenya, Rwanda, South Sudan, Tanzania, Uganda, 'policy', 'plan', 'strategy', 'guideline' and 'programme'. Additional key words included non-communicable disease, NCD, NCDs, HIV, HIV/AIDS, diabetes, hypertension and cardiovascular disease. Government websites for each country and bibliographies of relevant publications were also
Box 1 Information extracted from reviewed publications
1. Country.
2. Disease.
3. Title of strategy/guideline.
4. Author/s.
5. Date of publication.
6. Period of relevance.
7. The main goal of strategy/guideline.
8. Any action plan for HIV/NCD integration?
9. What services are being integrated?
10. Level of healthcare delivery where integration will occur?
11. Key quotes from the document.

searched. The retrieved documents were assessed for their authenticity and relevance based on the publishing organisation, language, date of publication and their period of relevance.

\section{Data extraction and analysis}

We developed a data extraction template based on the main research question. The headings in the template were based on a series of questions created to interrogate the document (box 1). The retrieved documents were manually searched for reference/s to the integration of services for HIV and NCDs. Any aspects of screening, prevention, treatment and control of HIV/AIDS into NCD services or vice versa were included. Documents describing intentions to deliver joint programmes to address HIV/AIDS and NCDs were also searched. Words such as 'integrate/integration', 'joint' and 'collaborate/ collaborative' were assumed to refer to the same intentions. The data templates were populated for each of the documents retrieved and relevant quotes to highlight specific points were extracted.

The extracted information was analysed using document analysis. This is a recognised qualitative, social research method whereby documents are systematically evaluated to generate empirical knowledge and develop understanding of a phenomenon. ${ }^{44}$ The analysis is presented as a narrative summary.

No patient or public involvement was possible.

\section{RESULTS}

A total of 31 documents that met our inclusion criteria, and were published between 2011 and 2020, were screened during the first stage of this review. The screened documents (table 1 ) included 14 policies, strategies or guidelines on HIV/AIDS, 9 documents on NCDs and 8 general health policies. Five documents were only available in French and were reviewed both by a native French speaker and using online translation tools.

Of the 31 documents screened, 13 contained action plans relating to the integration of HIV and NCD services. They were published from four countries and included four HIV/AIDS strategic plans, three HIV guidelines, two NCD strategic plans, one NCD treatment guideline, one 


\begin{tabular}{|c|c|c|c|}
\hline Country/region & $\begin{array}{l}\text { Focus of } \\
\text { document }\end{array}$ & Title & $\begin{array}{l}\text { Any action plan for } \\
\text { HIV/NCD integration? }\end{array}$ \\
\hline \multirow[t]{6}{*}{ Burundi } & General & Burundi Vision 2025, (2012-2025), ${ }^{69}$ & No \\
\hline & General & National Health Policy $2016-2025^{58}$ & No \\
\hline & HIV/AIDS & National Guidelines for HIV Prevention and Treatment in Burundi, $2020^{59}$ & No \\
\hline & HIV/AIDS & $\begin{array}{l}\text { Implementation Plan for National Guidelines for HIV Prevention and Treatment, } \\
2020^{60}\end{array}$ & No \\
\hline & NCD & $\begin{array}{l}\text { National Strategic Plan for the Control of Chronic Non-Communicable Diseases } \\
(2011-2015)^{64}\end{array}$ & No \\
\hline & NCD & Chronic Non-Communicable Disease Activity Plan, $2017^{65}$ & No \\
\hline \multirow[t]{4}{*}{ Kenya } & General & Kenya Health Policy, (2014-2030), ${ }^{45}$ & Yes \\
\hline & General & Kenya Health Sector Strategic Plan $(2018-2023)^{46}$ & No \\
\hline & NCD & $\begin{array}{l}\text { Kenya National Strategy for the Prevention and Control of Non-Communicable } \\
\text { Diseases, }(2015-2020),{ }^{70}\end{array}$ & Yes \\
\hline & NCD & Kenya National Guidelines for Cardiovascular Diseases Management $2018^{71}$ & Yes \\
\hline \multirow{2}{*}{$\begin{array}{l}\text { Republic of South } \\
\text { Sudan }\end{array}$} & General & South Sudan National Health Policy (2016-2026), ${ }^{67}$ & No \\
\hline & HIV/AIDS & $\begin{array}{l}\text { Republic of South Sudan, Country Operational Plan (COP) 2018, Strategic } \\
\text { Direction, - HIV/AIDS (2018), }{ }^{72}\end{array}$ & No \\
\hline \multirow[t]{3}{*}{ Rwanda } & General & Fourth Health Sector Strategic Plan, July 2018 - June $2024,{ }^{73}$ & No \\
\hline & HIV/AIDS & $\begin{array}{l}\text { Rwanda National Strategic Plan for HIV and AIDS } 2013 \text { - 2018; Extension } 2018 \text { - } \\
\text { 2020, }{ }^{74}\end{array}$ & Yes \\
\hline & HIV/AIDS & National HIV/AIDS Targets (2018-2030), ${ }^{54}$ & No \\
\hline \multirow{2}{*}{ Tanzania } & HIV/AIDS & $\begin{array}{l}\text { Operational manual for comprehensive differentiated delivery of HIV and AIDS } \\
\text { services, } 2019^{50}\end{array}$ & Yes \\
\hline & NCD & $\begin{array}{l}\text { Strategic and Action Plan for The Prevention and Control of Non-Communicable } \\
\text { Diseases in Tanzania, (2016-2020), }\end{array}$ & Yes \\
\hline \multirow[t]{4}{*}{ Uganda } & General & Uganda One Health Strategic Plan, (2018-2022), ${ }^{75}$ & No \\
\hline & HIV/AIDS & National HIV and AIDS Strategic Plans, $(2016-2020),{ }^{53}$ & Yes \\
\hline & HIV & Consolidated Guidelines for Prevention and Treatment of HIV in Uganda, $2020^{52}$ & Yes \\
\hline & NCD & The Uganda NCD Alliance Strategic Plan DRAFT (2016-2019) ${ }^{76}$ & No \\
\hline \multirow{2}{*}{$\begin{array}{l}\text { East African } \\
\text { Community }\end{array}$} & NCD & The East Africa NCD Charter $(2018)^{61}$ & No \\
\hline & NCD & $\begin{array}{l}\text { East Africa NCD Alliance Initiative. A Civil Society Benchmark Report. Responses } \\
\text { to NCDs in East Africa, } 2014^{62}\end{array}$ & Yes \\
\hline
\end{tabular}

NCD, non-communicable disease.

NCD charter and one general health policy. All documents reviewed were final versions except for the Uganda National HIV and AIDS Strategic Plan which was only found as a draft document.

The proposed actions to integrate the management of HIV/AIDS and NCD services identified through the document analysis are categorised according to the three approaches to HIV-NCD integration described by Duffy et $a l .{ }^{32}$

\section{NCD care integrated into HIV services}

The Kenyan National Health Policy ${ }^{45}$ and Health Sector Strategic $\mathrm{Plan}^{46}$ identify the care and management of HIV and NCDs as priority areas for intervention and investment. The updated Health Sector Strategic Plan outlined a commitment to achieving Universal Health Coverage for Kenya by 2022. The objectives outlined in the plan include, among others, enhancing efficiency and improving the availability of essential health services and 
using an integrated people-centred approach to service delivery. Several flagship projects are outlined as ways of achieving Universal Health Coverage including community high-impact interventions, a component of which is integrated community case management. However, little detail is provided of how these objectives will be achieved, and there is a recognition that the implementation of the Kenya Essential Package of Services and indeed other innovative service delivery strategies has been limited.

In the Kenyan HIV Treatment Guidelines, although not explicitly described as integrated management of HIV and NCDs, provides detail on lifestyle and drug management for HIV patients with diabetes and/or hypertension that can be implemented in the HIV clinic. ${ }^{47}$ A differentiated service delivery model where patients can access care at different levels is outlined, however, details on whether the 'integrated' management of HIV patients with comorbid NCDs can be managed at this level is not available.

There is a recognition in the Kenyan National AIDS Control Council Strategic framework that there needs to be better provision of HIV services, integrated into the essential health package and delivered in primary care. ${ }^{48}$

One of the objectives of this Strategic Framework is to 'increase coverage of care and treatment and reduce the loss from care' for people living with HIV in Kenya, to be achieved through the:

provision of screening and diagnostic equipment for TB, NCDs, malnutrition, opportunistic infections alongside those for HIV. ${ }^{48}$

Access to care and treatment at community and other non-antiretroviral therapy service points will be increased through integrated and decentralised HIV delivery models'. Health workers will be given 'integrated HIV trainings' and care givers will be provided with HIV education, literacy and empowerment'.

The Tanzanian HIV/AIDS management guideline recommends an integrated approach to community testing strategies that may include HIV, STI, tuberculosis (TB), blood glucose, blood pressure and nutrition assessments. ${ }^{49}$ Screening for hypertension, diabetes and dyslipidaemias when patients enrol in HIV care is recommended, as is ongoing monitoring and management of these conditions in the HIV clinic. The Tanzania HIV/ AIDS treatment guidelines refer to other documents for the detailed management of hypertension and diabetes.

Differentiated antiretroviral therapy delivery for stable clients, which includes those with controlled comorbidities, is outlined in this document however greater detail is provided in the Tanzanian Operational Manual for the Comprehensive Differentiated Delivery of HIV and AIDS Services (2019), which states the following:

Integration of services (TB/HIV, SRH/MNCH/PMTCT or NCD/HIV) aims at improving retention in care and reducing missed opportunities to provide comprehensive health services to clients requiring treatment for more than one condition. ${ }^{50}$
This integration is further described as a one-stop service for the stable HIV client, that is, a client whose diabetes or hypertension is controlled, occurring in the same consultation by the same healthcare provider and where NCD drug delivery is aligned with delivery of antiretroviral therapy. It is recognised that there are challenges to the delivery of integration including that if services are not available at a facility, integration cannot be achieved, integration requires communication between departments, adequately trained staff and consolidation of monitoring and evaluation tools. It is not clear, however, if this integrated model can be extended to antiretroviral therapy delivery models that are community based.

The Ugandan National Health Policy recognises the growing burden of HIV and NCDs, and has as one of its guiding principles that,

Curative, preventive and promotive services shall be provided in an integrated manner. ${ }^{51}$

The 2020 Uganda Consolidated Guidelines for the Prevention and Treatment of HIV and AIDS provides a clear statement supporting integrated management of HIV and NCDs by recommending that stable HIV patients with NCDs and without any complications should be seen in a single appointment by the same clinician (where possible), with patient records available in the same location, and that both HIV and NCD health education should be available in the clinic. ${ }^{52} \mathrm{~A}$ differentiated service delivery model is also outlined. However, patients with multimorbidity or with uncontrolled chronic comorbidities are considered to be complex or unstable, and therefore, not eligible for community-based care. Whether these patients could receive community-based care once control of comorbidities is achieved is not clear.

The goal of the Uganda HIV/AIDS strategy (20162020) is to move towards halting HIV incidence, HIV and AIDS-related mortality and morbidity and HIVrelated discrimination. ${ }^{53}$ One of the strategic actions to strengthen integration of HIV care and treatment within healthcare programmes is to:

Integrate HIV care and treatment with maternal, newborn and child health, sexual and reproductive health and rights, mental health and non-communicable / chronic diseases. (p.27)

The proposed integration would occur across all levels including community and home-based care. No further details about the plan are outlined in the strategy.

In Rwanda the HIV Strategic Plan and HIV Targets outlines a focus on better integration of HIV services within broader health programmes, although NCDs are not mentioned specifically. ${ }^{54}$ They state that integration of HIV services into the health system has always been a strong characteristic of the Rwandan HIV response, and this has benefited both the HIV programme and the health system in general. There is also a recognition of the regional integration of the HIV response with 
the East African Community and the need to establish harmonised protocols and guidelines for HIV prevention and care interventions in all countries of the region. The HIV Strategic Plan addresses NCDs by ensuring access to screening, diagnosis and treatment for people living with HIV, increasing diagnostic capacity, and improving healthcare workers capacity, as part of a strategy for decreasing morbidity and mortality of people living with HIV.

In Rwanda, apart from discussion within the context of differentiated service delivery, the integrated screening or management of NCDs in HIV patients is not mentioned. ${ }^{556}$ Patients who have HIV and NCDs are initially described as being ineligible for differentiated service delivery until they have stabilised. Whether care can then be delivered in the community for both conditions is not clear.

The Rwanda NCD National Strategic Plan identifies several challenges to the delivery of NCD-related care, including that there is a lack of internal reliable data about the burden of disease for NCDs that might have informed a strong evidence-based approach for planning.${ }^{57}$ Provision of NCD services is limited, and a priority of the National Strategic Plan is to raise awareness of the service to generate demand. HIV is not recognised as a risk factor for the development of diabetes.

In Burundi in 2011, a merger of the Ministries of Health and HIV enabled the integration of AIDS programmes into the general healthcare package, and a priority action area is the integration of HIV, TB and Malaria programmes. ${ }^{58}$ Integration of HIV and NCD programmes are not explicitly mentioned. The Burundi National Guidelines for the Prevention and Treatment of HIV discuss the clinical considerations of managing patients with diabetes and HIV comorbidity, and recommend annual blood sugar measurements, however, there is no outlined plan for screening of NCDs or recognition of increased risk in the HIV patient group. ${ }^{59}$ The strengthening of integration of HIV care with TB, reproductive health, maternal and child health and secondary care is recommended. The Implementation Plan associated with these guidelines does not outline any assessment of NCD prevalence or risk factors, although in describing education provision to patients recommends that "under certain conditions, additional steps can be added including; common information on chronic diseases ${ }^{60}$

On a regional note, the East Africa NCD Charter, published by the EAC Alliance, proposes the development of strategic plan to address the growing burden of NCDs in the region. ${ }^{61}$ The focus is to mainstream NCDs and health into all national development plans through policy coherence and coordinated action. It does not outline any country specific action plans or focus on integration. In a review of policy progress on NCDs in the East African Community, it was noted that health systems needed to be strengthened to address NCDs, and that there was limited implementation of existing NCD related policies in the region. ${ }^{62} \mathrm{~A}$ recommendation from this review was that NCD prevention and control activities needed to be integrated into existing health platforms, including those for HIV care.

\section{HIV care services added to NCD prevention and control}

The Kenya health policy reflects the country's effort to attain the highest possible standard of health in response to the needs of the population. ${ }^{45}$ It aims to include prevention and control of NCDs into established communicable disease programmes. The purpose is to 'leverage on' the country's existing communicable diseases management capacity. This overarching national health policy objective is further reflected in the Kenya national strategy for the prevention and control of NCDs (2015-2020). The strategy proposes;

integrating non-communicable disease control into communicable disease prevention and control using existing primary health care platforms such as community health services, maternal and child health, school health, disease surveillance, HIV, TB and Malaria as entry points (p.15).

No further details about the integration, or mainstreaming process are given in the strategy, although one of the performance indicators is the "number of health programmes incorporating NCD prevention and control'.

In Tanzania's NCD strategy, a guiding principle is that an integrated approach, taking account of the shared characteristics of several diseases and focusing on functions rather than on diseases, should be favoured. ${ }^{63}$ The strategy aims to achieve a $20 \%$ reduction in the morbidity, disability and premature mortality from NCDs by 2020 , compared with levels in 2016 , by applying a patient-centred model of delivery to integrate NCD care into existing services, and making NCD prevention and control a national priority. A key action is the establishment of collaborative TB, diabetes and HIV services, however, there is little mention of integrating into general HIV care.

The Rwanda NCD plan (2014-2019) targets an 80\% reduction in NCD related mortality for people under the age of 40 over a 5 -year period, $2015-2020 .^{57}$ To improve early detection of NCDs, the strategy aims to raise awareness of risk factors for NCDs, available healthcare facilities and screening programmes, and to decentralise NCD care to provincial and district levels. In this document, reference to integration of HIV and NCD services was made with regards to the training of community health workers. Over the lifetime of the strategy, two community health workers from each village will be trained in chronic and palliative care. This will include 'adherence support for advanced chronic conditions such as HIV, heart failure, insulin-dependent diabetes and cancers'. They will be trained in group-based psychotherapy techniques and equipped to provide home based support to patients suffering from chronic conditions.

In Burundi the National Strategic Plan for Control of NCDs includes as a priority area of intervention that 
chronic NCD care should be integrated at all levels of care and recognises that there are insufficient resources available for prevention, diagnosis and treatment of NCDs. ${ }^{64}$ There is recognition of the growing burden of NCDs as an emerging health threat, and that there is a lack of available information on the prevalence of NCDs in the country. The National Integrated NCD Control Programme Activity Plan mentions coordination of interventions in the fight against chronic NCDs, however, neither of these documents mention integrated screening or treatment of NCDs and HIV. ${ }^{65}$

\section{Combined approach to tackle chronic conditions including NCDS and HIV/AIDS}

The high-level national health policies in all the countries included, outline plans to increase access to care across HIV and NCD programmes. In Kenya there is a commitment to achieving Universal Health Coverage and improving access to person-centred essential health services. ${ }^{46}$ Uganda's Second National Health Policy states that 'Curative, preventive and promotive services shall be provided in an integrated manner', although there is little detail provided. ${ }^{51}$ Tanzania's Health Sector strategic plan describes Integrated delivery of a reviewed package of essential healthcare interventions... decentralised to the facility level ${ }^{66}$ South Sudan's National Health Policy has the delivery of Universal Health Coverage as a policy objective. ${ }^{67}$

The Burundi National Health Policy outlines a plan to work towards Universal Health Coverage and includes as one of its guiding principles 'continuity, integration, acceptability and quality of care ${ }^{58}$ In Kenya and Tanzania specific combined approaches to target HIV and NCDs are mentioned in relation to integrated screening programmes. ${ }^{48} 49$

\section{DISCUSSION}

This review shows that integrated delivery of HIV and NCD care is recommended in high level health policies and treatment guidelines in four East African countries; Kenya, Rwanda, Tanzania and Uganda. Most of this relates to integrating NCD prevention and control into existing HIV programmes. Similarly, three documents had plans to incorporate prevention and control of HIV into existing NCD services.

Evidence on the effectiveness of the 'adding on' model of integrating NCD and HIV services in low-income and middle-income countries, is limited and while this may lead to financial savings and improved service utilisation, it may not lead to improved patient outcomes. ${ }^{3}$ In Tanzania, Uganda and Kenya, the most recent HIV and AIDS treatment guidelines recommend the delivery of an integrated service for patients who have HIV and uncomplicated NCDs, where care for these conditions is delivered in the same consultation and there is alignment of drug delivery and record keeping. This is a recent change in policy, having been included in guidelines issued within the last year, and specifically appear to apply to differentiated service delivery models in primary care.
Evidence for the proposed actions were not outlined in the strategies or guidelines. While it is clear that HIV care can be delivered in a variety of community-based settings, it is not clear if integrated HIV and NCD care can be delivered outside of a health facility.

It is possible that these countries have taken steps to integrate NCD and HIV prevention and control as a pragmatic approach to address the growing burden of disease from HIV and NCDs, particularly as external funding for HIV treatment programmes continues to decline. The differences between countries in the adoption and development of an integrated service delivery model is likely to also reflect the capacity of different countries to deliver this. Both South Sudan and Burundi report a focus on building their healthcare infrastructure and improving coverage and quality of care provision. The Rwanda NCD Strategic plan describes a nascent NCD service where comprehensive NCD services only started in 2005. In Burundi, NCDs are described as an emerging health threat, with little available information on the burden. In these countries capacity and coverage of NCD services is limited, making integration with HIV care difficult to implement. ${ }^{6}$ Burundi, South Sudan and Rwanda have lower HIV prevalence among adults aged $15-49$ years $(0.9 \%, 2.5 \%$ and $2.6 \%$, respectively) than Kenya, Tanzania and Uganda $(4.5 \%, 4.8 \%$ and $5.8 \%$, respectively) ${ }^{68}$ The larger HIV programmes in countries with higher prevalence may present a greater opportunity for expanding NCD care provision, as reflected in their policies.

There is a growing evidence base that integration is feasible, and now global high level support for this policy direction and recognition of the role this could play in achieving Universal Health Coverage. ${ }^{24}$ New targets from UNAIDS aimed at helping to achieve the Sustainable Development Goals, includes targets focused on peoplecentred, context-specific service integration, where at least $90 \%$ of people living with HIV and individuals at heightened risk of HIV infection are linked to services for other communicable diseases, NCDs, sexual and genderbased violence, mental health and other services they need for their overall health and well-being, ${ }^{24}$ How this is to be achieved is not clear, and the response in each country will be dependent on the available resources and epidemiology of each condition. Further research is needed on different integration options and strategies for delivery across settings and patient groups, taking into account both provider and patient perspectives.

\section{CONCLUSION}

Despite the limited evidence of effectiveness, several countries have taken steps to integrate services for NCDs and HIV, mainly by adding NCD prevention and control into HIV care delivery. Should integration prove to be effective, there will be increased coverage of NCD services and potential improvements in health outcomes for those with NCDs. However, with recent reductions in 
HIV programme funding there is a significant risk that integration could spread resources too thinly, jeopardising the success of HIV services, resulting in worse health outcomes for people living with HIV and little benefit for those living with NCDs. High-quality and timely monitoring and evaluation of the proposed initiatives, including of patient level clinical outcomes, is essential. This will generate evidence on the benefits, challenges and cost-effectiveness of integration of HIV and NCD services and will be particularly useful for those countries where NCD services are still developing.

\section{Author affiliations}

${ }^{1}$ Department of International Public Health, Liverpool School of Tropical Medicine, Liverpool, UK

${ }^{2}$ MRC/UVRI and LSHTM Uganda Research Unit, Entebbe, Uganda

${ }^{3}$ Department of Internal Medicine and Specialties, Faculty of Medicine and Biomedical Sciences, University of Yaounde 1, Yaounde, Cameroon

${ }^{4}$ Muhimbili Medical Research Centre, National Institute of Medical Research, Dar es Salaam, United Republic of Tanzania

${ }^{5}$ Shree Hindu Mandal Hospital, Dar es Salaam, United Republic of Tanzania ${ }^{6}$ Department of Clinical Sciences, Liverpool School of Tropical Medicine, Liverpool, UK

Contributors $A G$ conceptualised this review and provided overall supervision. $\mathrm{OA}$, $A G, S J$ and TN were involved in the design of this review. $O A$ and $A G$ undertook the literature search (assisted by KN and JCM for French documents), screening, data extraction, data analysis and production of successive versions of the manuscript. AG, SJ and ML critically reviewed and edited successive versions of the paper. ML, $J B, S M$ and KR provided further critical review of the manuscript.

Funding The authors have not declared a specific grant for this research from any funding agency in the public, commercial or not-for-profit sectors.

Competing interests None declared.

Patient and public involvement Patients and/or the public were not involved in the design, or conduct, or reporting, or dissemination plans of this research.

Patient consent for publication Not required.

Ethics approval This review was not formally submitted to an ethical review board, as it relied on publicly available data.

Provenance and peer review Not commissioned; externally peer reviewed.

Data availability statement № data are available. All the information used in this paper is publically available.

Open access This is an open access article distributed in accordance with the Creative Commons Attribution Non Commercial (CC BY-NC 4.0) license, which permits others to distribute, remix, adapt, build upon this work non-commercially, and license their derivative works on different terms, provided the original work is properly cited, appropriate credit is given, any changes made indicated, and the use is non-commercial. See: http://creativecommons.org/licenses/by-nc/4.0/.

ORCID iD

Shabbar Jaffar http://orcid.org/0000-0002-9615-1588

\section{REFERENCES}

1 Bloomfield GS, Khazanie P, Morris A, et al. Hiv and noncommunicable cardiovascular and pulmonary diseases in lowand middle-income countries in the art era: what we know and best directions for future research. J Acquir Immune Defic Syndr 2014;67 Suppl 1:S40-53.

2 Deeks SG, Lewin SR, Havlir DV. The end of AIDS: HIV infection as a chronic disease. Lancet 2013;382:1525-33.

3 Dudley L, Garner P. Strategies for integrating primary health services in low- and middle-income countries at the point of delivery. Cochrane Database Syst Rev 2011;7:CD003318.

4 Haldane V, Legido-Quigley H, Chuah FLH, et al. Integrating cardiovascular diseases, hypertension, and diabetes with HIV services: a systematic review. AIDS Care 2018;30:103-15.
5 Soto TA, Bell J, Pillen MB, et al. Literature on integrated HIV care: a review. AIDS Care 2004;16 Suppl 1:S43-55.

6 Matanje Mwagomba BL, Ameh S, Bongomin P, et al. Opportunities and challenges for evidence-informed HIV-noncommunicable disease integrated care policies and programs: lessons from Malawi, South Africa, Swaziland and Kenya. AIDS 2018;32 Suppl 1:S21-32.

7 World Health Organization. Hiv/Aids Factsheet. Geneva: World Health Organization, 2019. https://www.who.int/news-room/factsheets/detail/hiv-aids

8 El-Sadr WM, Goosby E. Building on the HIV platform: tackling the challenge of noncommunicable diseases among persons living with HIV. AIDS 2018;32 Suppl 1:S1-3.

9 Palmisano L, Vella S. A brief history of antiretroviral therapy of HIV infection: success and challenges. Ann Ist Super Sanita 2011;47:44-8.

10 Harrison KM, Song R, Zhang X. Life expectancy after HIV diagnosis based on national HIV surveillance data from 25 states, United States. J Acquir Immune Defic Syndr 2010;53:124-30.

11 World Health Organization. Antiretroviral therapy coverage Estimates by WHO region 2019, 2019. Available: http://apps.who.int/ gho/data/view.main.23300REGION?lang=en

12 World Health Organization. Non communicable diseases factsheet, 2018. Available: https://www.who.int/news-room/fact-sheets/detail/ noncommunicable-diseases [Accessed 12 Dec 2019].

13 World Health Organization. Global status report on noncommunicable diseases, 2010.

14 Addo J, Smeeth L, Leon DA. Hypertension in sub-Saharan Africa: a systematic review. Hypertension 2007:50:1012-8.

15 Asmelash D, Asmelash Y. The burden of undiagnosed diabetes mellitus in adult African population: a systematic review and metaanalysis. J Diabetes Res 2019;2019:4134937.

16 Yu D, Souteyrand Y, Banda MA, et al. Investment in HIV/AIDS programs: does it help strengthen health systems in developing countries? Global Health 2008;4:8.

17 Marsh K, Eaton JW, Mahy M, et al. Global, regional and country-level 90-90-90 estimates for 2018: assessing progress towards the 2020 target. AIDS 2019;33 Suppl 3:S213-26.

18 Atun R, Davies JI, Gale EAM, et al. Diabetes in sub-Saharan Africa: from clinical care to health policy. Lancet Diabetes Endocrinol 2017;5:622-67.

19 Labhardt ND, Balo J-R, Ndam M, et al. Improved retention rates with low-cost interventions in hypertension and diabetes management in a rural African environment of nurse-led care: a cluster-randomised trial. Trop Med Int Health 2011;16:1276-84.

20 Musicha C, Crampin AC, Kayuni N, et al. Accessing clinical services and retention in care following screening for hypertension and diabetes among Malawian adults: an urban/rural comparison. $J$ Hypertens 2016;34:2172-9.

21 Waddington C, Egger D. Integrated Health Services - What and Why? Geneva, Switzerland, 2008.

22 Briggs CJ, Garner P. Strategies for integrating primary health services in middle- and low-income countries at the point of delivery. Cochrane Database Syst Rev 2006;2:CD003318.

23 Bekker L-G, Alleyne G, Baral S, et al. Advancing global health and strengthening the HIV response in the era of the sustainable development goals: the International AIDS Society-Lancet Commission. Lancet 2018:392:312-58.

24 UNAIDS. Prevailing against pandemics by putting people at the centre. world AIDS day report. Geneva, Switzerland: UNAIDS, 2020

25 United Nations. Sustainable development goals knowledge platform: United nations, 2020. Available: https://sustainabledevelopment.un. org/?menu=1300 [Accessed 10 Jan 2020].

26 Haregu TN, Setswe G, Elliott J, et al. Integration of HIV/AIDS and noncommunicable diseases in developing countries: rationale, policies and models. Int $J$ Healthc 2015;1:21-7.

27 UNAIDS. Chronic care of HIV and noncommunicable diseases Geneva. UNAIDS, 2011

28 United Nations. Political Declaration on HIV and AIDS: on the fast track to accelerating the fight against HIV and to ending the AIDS epidemic by 2030: United nations, 2016. Available: https://www. unaids.org/sites/default/files/media_asset/2016-political-declarationHIV-AIDS en.pdf

29 Samji H, Cescon A, Hogg RS, et al. Closing the gap: increases in life expectancy among treated HIV-positive individuals in the United States and Canada. PLoS One 2013:8:e81355

30 AVERT. Funding for HIV and AIDS: avert, 2019. Available: https:// www.avert.org/professionals/hiv-around-world/global-response/ funding\#footnote11 9sh6woi [Accessed 10 Jan 2020].

31 UNAIDS. Prevention gap report Geneva, Switzerland: UNAIDS, 2016. Available: https://www.unaids.org/sites/default/files/media asset/ 2016-prevention-gap-report_en.pdf 
32 Duffy M, Ojikutu B, Andrian S, et al. Non-Communicable diseases and HIV care and treatment: models of integrated service delivery. Trop Med Int Health 2017;22:926-37.

33 Rabkin M, de Pinho H, Michaels-Strasser S, et al. Strengthening the health workforce to support integration of HIV and noncommunicable disease services in sub-Saharan Africa. AIDS 2018;32 Suppl 1:S47-54.

34 Garrib A, Birungi J, Lesikari S, et al. Integrated care for human immunodeficiency virus, diabetes and hypertension in Africa. Trans $R$ Soc Trop Med Hyg 2019;113:809-12.

35 Edwards JK, Bygrave H, Van den Bergh R, et al. Hiv with noncommunicable diseases in primary care in Kibera, Nairobi, Kenya: characteristics and outcomes 2010-2013. Trans R Soc Trop Med Hyg 2015;109:440-6.

36 Golovaty I, Sharma M, Van Heerden A, et al. Cost of integrating noncommunicable disease screening into home-based HIV testing and counseling in South Africa. J Acquir Immune Defic Syndr 2018;78:522-6.

37 Achwoka D, Waruru A, Chen T-H, et al. Noncommunicable disease burden among HIV patients in care: a national retrospective longitudinal analysis of HIV-treatment outcomes in Kenya, 20032013. BMC Public Health 2019;19:372.

38 Chamie G, Kwarisiima D, Clark TD, et al. Leveraging rapid community-based HIV testing campaigns for non-communicable diseases in rural Uganda. PLoS One 2012;7:e43400.

39 Kagaruki GB, Mayige MT, Ngadaya ES, et al. Magnitude and risk factors of non-communicable diseases among people living with HIV in Tanzania: a cross sectional study from Mbeya and Dar ES Salaam regions. BMC Public Health 2014;14:904.

40 Wroe EB, Kalanga N, Mailosi B, et al. Leveraging HIV platforms to work toward comprehensive primary care in rural Malawi: the integrated chronic care clinic. Healthc 2015;3:270-6.

41 World Health Organization. A framework for national health policies, strategies and plans Geneva. Switzerland: World Health Organization, 2010. http://www.euro.who.int/_data/assets/pdf_file/ 0008/120995/RC60_etechdoc2.pdf

42 East African Community. Quick facts about EAC Arusha, Tanzania, 2019. Available: https://www.eac.int/eac-quick-facts2019

43 The World Bank. World bank country and lending groups, 2019. Available: https://datahelpdesk.worldbank.org/knowledgebase/ articles/906519-world-bank-country-and-lending-groups2019

44 Bowen GA. Document analysis as a qualitative research method. Qualitative Research Journal 2009;9:27-40.

45 Kenya Ministry of Health. Kenya health policy 2014 - 2030 Nairobi. Kenya: Ministry of Health, 2014. https://www.afidep.org/?wpfb_dl= 80

46 Kenya Ministry of Health. Kenya health sector strategic plan. transforming health systems: achieving universal health coverage by 2022. Ministry of Health, 2018.

47 Kenya Ministry of Health. Guidelines on use of antiretroviral drugs for treating and preventing HIV in Kenya. Kenya: Ministry of Health, 2018.

48 Kenya Ministry of Health. Kenya AIDS Strategic Framework 2014/2015 - 2018/2019 Nairobi, Kenya: Ministry of Health, 2015. http://nacc.or.ke/wp-content/uploads/2015/09/KASF_Final.pdf

49 United Republic of Tanzania National AIDS Control Programme. National guidelines for the management of HIV and AIDS. Tanzania: Ministry of Health cD, Gender, Elderly and Children, 2019.

50 United Republic of Tanzania National AIDS Control Programme. Operational manual for comprehensive differentiated delivery of HIV and AIDS services. Tanzania: Minisstry of Health CD, Gender, Elderly and Children, 2019.

51 Republic of Uganda ministry of Health. The second National health policy. promoting peoples health to enhance socio-economic development. Kampala, Uganda: Ministry of Health, 2010.

52 Republic of Uganda Ministry of Health. Consolidated guidelines for the prevention and treatment of HIV and AIDS in Uganda. Uganda: Ministry of Health, 2020.

53 The Uganda AIDS Commission. National HIV and AIDS strategic plan 2015/2016-2019/2020, an AIDS free Uganda, my responsibility! Uganda: The Uganda AIDS Commission, 2015. http://www.ilo.org/ wcmsp5/groups/public/-ed_protect/-protrav/-ilo_aids/documents/ policy/wcms_455260.pdf

54 Republic of Rwanda Ministry of Health. Rwanda National HIV/AIDS Targets 2018 - 2020 - 2030 Rwanda: Ministry of Health, 2015. Available: https://www.childrenandaids.org/sites/default/files/ 2018-05/Rwanda_HIV\%20AIDS\%20Targets\%202018-2020-2030. pdf
55 Republic of Rwanda Rwanda Biomedical Centre. National guidelines for prevention and management of HIV and STIs. Rwanda: Ministry of Health, 2016.

56 Republic of Rwanda Rwanda Biomedical Center. Circular of key changes in HIV prevention and management guidelines. Kigali, Rwanda: Ministry of Health, Republic of Rwanda, 2018.

57 Republic of Rwanda Ministry of Health. Rwanda Noncommunicable Diseases National Strategic Plan 2014 - 2019. Rwanda: Ministry of Health, 2019. https://www.iccp-portal.org/system/files/plans/RWA B3_NCD_NSP_strategic_plan_2014-2019_v12.pdf

58 Republic of Burundi Ministry of Public Health and the fight against AIDS. National Health Policy 2016 - 2025. Burundi: AIDS MoPHatfa, 2016.

59 Republic of Burundi Ministry of Public Health and the fight against AIDS. National guidelines for the prevention and treatment of HIV in Burundi. Bujumbura, Burundi: Ministry of Public Health and the fight against AIDS, 2020.

60 Republic of Burundi Ministry of Public Health and the fight against AIDS. Implementation plan for national guidelines for HIV prevention and treatment. Bujumbura, Burundi: Ministry of Public Health and the fight against AIDS, 2020.

61 East Africa NCD Alliance Initiative. The East Africa Ncd charter. accelerating the fight against NCDS in East Africa. Kigali, Rwanda, 2018.

62 East Africa NCD Alliance Initiative. Responses to NCDS in East Africa. A civil society benchmark report, 2014.

63 United Republic of Tanzania Ministry of Health. Strategic and Action Plan for the Prevention and Control of Non-communicable Diseases in Tanzania 2016 - 2020. Tanzania: Ministry of Health, Community Development, Gender, Elderly and Children, 2016. https://www. worlddiabetesfoundation.org/sites/default/files/NCD\%20Stategic\% 20Plan\%202016\%20-\%202020.pdf

64 Republic of Burundi Ministry of Public Health and the fight against AIDS. National strategic plan for the control of chronic noncommunicable diseases (2011-2015. Ministry of Public Health and the fight against AIDS, 2011.

65 Republic of Burundi Ministry of Public Health and the fight against AIDS. National integrated chronic non-communicable disease control programme activity plan 2017. Burundi, 2016.

66 United Republic of Tanzania Ministry of Health and Social Welfare. Health Sector Strategic Plan, 2015 - 2020 Tanzania, 2015. Available: http://www.tzdpg.or.tz/fileadmin/documents/dpg_internal/dpg_ working_groups_clusters/cluster_2/health/Key_Sector_Documents/ Induction Pack/Final HSSP IV Vs1.0 260815.pdf

67 Republic of South Sudan Ministry of Health. National health policy 2016-2026 Republic of South Sudan: Ministry of health, 2016. http:// www.nationalplanningcycles.org/sites/default/files/planning_cycle_ repository/south_sudan/south_sudan_national_health_policy_2016_ to_2025_2.pdf

68 UNAIDS. Countries: UNAIDS, 2021. Available: https://www.unaids. org/en/regionscountries/countries [Accessed 20 Mar 2021].

69 Republic of Burundi Ministry of Planning and Communal Development and United Nations Development Programme in Burundi. Vision Burundi 2025. Burundi, 2011.

70 Kenya Ministry of Health. Kenya National Strategy for the Prevention and Control of Non-communicable diseases 2015 - 2020. Nairobi, Kenya: Ministry of Health, 2015. https://www.who.int/nmh/ncd-taskforce/kenya-strategy-ncds-2015-2020.pdf

71 Kenya Ministry of Health. Kenya national guidelines for cardiovascular diseases management. Kenya: Diseases DoN-C 2018.

72 PEPFAR. Republic of South Sudan country operational plan (COP) 2018 strategic direction summary PEPFAR, 2018.

73 Republic of Rwanda Ministry of Health. Fourth health sector strategic plan Rwanda: Ministry of health, 2018. Available: https:// www.childrenandaids.org/sites/default/files/2018-05/Rwanda_Nat\% 20Health\%20Sector\%20Plan_2018-2024.pdf

74 Republic of Rwanda Ministry of Health. Rwanda National Strategic Plan for HIV and AIDS 2013 - 2018 Rwanda: Ministry of Health. Available: http://www.nationalplanningcycles.org/sites/default/files/ country_docs/Rwanda/final_nsp_2013-2018.pdf

75 Republic of Uganda One Health Platform. Uganda One Health Strategic Plan 2018 - 2022. Uganda: The National One Health Platform, 2018. http://health.go.ug/sites/default/files/Uganda\% 20OHSP\%20Final\%20Launched\%2015-02-2018\%20\%281\%29.pdf

76 Uganda NCD Alliance. The Uganda Ncd alliance strategic plan Kampala. Uganda: Uganda NCD Alliance, 2016. https:// ncdalliance.org/sites/default/files/Sample\%206-UNCDA\%20Draft\% 20Strategic\%20plan\%202016\%20-\%202019.pdf 